INPLASY

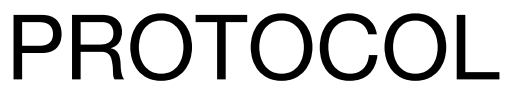

To cite: Li et al. Bayesian network meta-analysis analysis of PBA, HPB, DCB, DEB, CtB

for the Treatment of

Autogenous arteriovenous

fistula Stenosis. Inplasy

protocol 202190099. doi:

10.37766/inplasy2021.9.0099

Received: 27 September 2021

Published: 27 September 2021

Corresponding author:

Yu Li

15811480832@163.com

Author Affiliation:

Xuanwu Hospital, Capital

Medical University.

Support: None.

Review Stage at time of this submission: Data analysis -

Completed but not published.

Conflicts of interest:

None declared.

\section{Bayesian network meta-analysis analysis of PBA, HPB, DCB, DEB, CtB for the Treatment of Autogenous arteriovenous fistula Stenosis}

Li, Y1; Cui, W2; Wang, J3; Chen, X4; Zhang, C5; Luo, T6.

Review question / Objective: This study aimed to explore the effectiveness of angioplasty with different balloons in patients with AVF stenosis.

Eligibility criteria: 1. Studies included in the NMA were RCTs from database establishment to January 31, 2021. 2. Stenotic AVF was defined as stenosis $\geq 50 \%$ and the blood flow $(\mathrm{Qa})$ in fistula was less than $500 \mathrm{ml} / \mathrm{min}$, which could not meet the requirement of hemodialysis. 3 . There was no restriction with regard to publication status; however, language was confined to English and Chinese. 4. Studies were limited to human trials. Meanwhile, at least 3 months of follow-up. 5 . Restenosis of AVF was excluded from this study.

Information sources: PubMed, EMBASE, the Cochrane Central Register of Controlled Trials (CENTRAL), Scopus and ClinicalTrials.gov databases.

INPLASY registration number: This protocol was registered with the International Platform of Registered Systematic Review and Meta-Analysis Protocols (INPLASY) on 27 September 2021 and was last updated on 27 September 2021 (registration number INPLASY202190099).

\section{INTRODUCTION}

Review question / Objective: This study aimed to explore the effectiveness of angioplasty with different balloons in patients with AVF stenosis.
Condition being studied: Chronic kidney disease (CKD) is a condition of irreversible destruction of the renal parenchyma, with a progressive loss of kidney function over several years. Meanwhile, morbidity of CKD is gradually increasing in the past decades. Maintenance hemodialysis (MHD) has been 
recommended as the best alternative for renal transplant, in treatment of end stage renal disease (ESRD), due to shortage of donor organ. However, it is important to note that patients undergoing MHD need a patent vascular access (VA), which functions adequately for a long time and without complications. This is especially critical for the patients' survival and quality of life. Autogenous arteriovenous fistula (AVF) is the optimal vascular access for patients undergoing MHD and it is reflected in the Kidney Disease Outcomes and Quality Initiative (KDOQI) guidelines. AVF is constructed by subcutaneous anastomosis of an artery with an adjacent vein (mostly the radial artery and cephalic vein). However, the application of AVF may be limited by stenosis which accounts for intimal hyperplasia. Balloon angioplasty (BA) has been recommended for the treatment of AVF stenosis by ESVES European guidelines. The first widely adopted endovascular intervention for AVF stenosis was plain balloon angioplasty (PBA), which remains a common treatment. However, this treatment intervention is susceptible to acute vessel elastic recoil. Therefore, several treatment strategies have been proposed and tested, including high-pressure balloon (HPB), drug-coated balloon (DCB), drug eluting balloon (DEB) and cutting balloon (CtB). Previously, original data on the effect of these treatment strategies were reported from several randomized controlled trials (RCTs). Nevertheless, direct evidence from interventions evaluated directly in head-tohead trials is rare. Therefore, it still remains unclear whether treatment options involving new type of balloons can provide better outcomes. Therefore, the purpose of this study was to comprehensively review the effectiveness of different treatment options in patients with AVF stenosis. Six members of our subject group will jointly complete this study.

\section{METHODS}

Participant or population: Twenty randomized controlled trials (RCTs) involving 2242 participants were included in our study.
Intervention: High-pressure balloon (HPB), drug-coated balloon (DCB), drug eluting balloon (DEB) and cutting balloon (CtB).

Comparator: plain balloon angioplasty (PBA).

\section{Study designs to be included: RCT.}

Eligibility criteria: 1 . Studies included in the NMA were RCTs from database establishment to January 31, 2021. 2. Stenotic AVF was defined as stenosis $\geq$ $50 \%$ and the blood flow $(\mathrm{Qa})$ in fistula was less than $500 \mathrm{ml} / \mathrm{min}$, which could not meet the requirement of hemodialysis. 3 . There was no restriction with regard to publication status; however, language was confined to English and Chinese. 4. Studies were limited to human trials. Meanwhile, at least 3 months of follow-up. 5. Restenosis of AVF was excluded from this study.

Information sources: PubMed, EMBASE, the Cochrane Central Register of Controlled Trials (CENTRAL), Scopus and ClinicalTrials.gov databases

Main outcome(s): Primary patency rates of AVF at 3, 6, 9, and 12 months.

Quality assessment / Risk of bias analysis: Cochrane tools.

Strategy of data synthesis: The NMA was performed by STATA 15 (network and mvmeta commands) and GeMTC software. Fixed-effects model (Peto method) and Random-effects model (Mantel-Haenszel method) were used to analyze nonheterogeneous and heterogeneous data, respectively.

\section{Subgroup analysis: None.}

Sensitivity analysis: A certain study was removed each time, and a new metaanalysis was conducted separately to investigate whether the results changed or not. If the new results were inconsistent with the previous results, it is considered that this study has a great impact on the total effect. 
Country(ies) involved: China.

Keywords: Arteriovenous fistula, Network meta-analysis, Plain balloon angioplasty, High-pressure balloon, Drug coated balloon, Drug eluting balloon, Cutting balloon.

Contributions of each author:

Author 1 - Yu Li.

Author 2 - Wenhao Cui.

Author 3 - Jukun Wang.

Author 4 - Xin Chen.

Author 5 - Chao Zhang.

Author 6 - Tao Luo. 